PROCEEDINGS OF THE

AMERICAN MATHEMATICAL SOCIETY

Volume 133, Number 1, Pages 103-113

S 0002-9939(04)07498-2

Article electronically published on June 2, 2004

\title{
ON SMOOTHNESS OF SYMMETRIC MAPPINGS II
}

\author{
A. CANTÓN
}

(Communicated by Juha M. Heinonen)

\begin{abstract}
If the dilatation of a quasiconformal selfmap of the upper halfplane vanishes near the real line as a power of the height, the induced quasisymmetric mapping is Lipschitz with the same exponent. In this note, it is shown that the converse does not hold for any positive exponent. In addition, a sufficient condition is found to have locally a quasiconformal extension with the desired growth in the dilatation.
\end{abstract}

\section{InTRODUCTION AND NOTATION}

We will consider quasiconformal selfmappings $f$, of the upper half-plane $\mathbb{H}$, that fix $\infty$. These maps induce a boundary homeomorphism, $h$ on $\mathbb{R}$, that satisfies the $M$-condition, namely:

$$
\frac{1}{M} \leq\left|\frac{h(x+t)-h(x)}{h(x)-h(x-t)}\right| \leq M
$$

for all $t>0$ and $x \in \mathbb{R}$.

Conversely, if $h: \mathbb{R} \rightarrow \mathbb{R}$ is an increasing homeomorphism for which the $M$ condition holds (so-called quasisymmetric mapping), then there exists a quasiconformal extension of $h$ to the upper half-plane. This extension is not unique. In spite of the regularity implied by the $M$-condition, quasisymmetric mappings can be singular with respect to the Lebesgue measure (see [4]).

We shall use the standard notation for the derivatives of $f, f_{z}=\partial f / \partial z$ and $f_{\bar{z}}=\partial f / \partial \bar{z}$. The complex dilatation of a quasiconformal mapping $f$ at the point $z$ is $\mu_{f}(z)=f_{\bar{z}}(z) / f_{z}(z)$. This dilatation is defined almost everywhere and $\left\|\mu_{f}\right\|_{\infty}<1$. When $\left\|\mu_{f}\right\|_{\infty}=0, f$ is a conformal automorphism of $\mathbb{H}$.

We say that $f$ is locally quasiconformal if $\operatorname{ess}^{\sup _{z \in K}}\left|\mu_{f}(z)\right| \leq k<1$ for every compact set in $K \subset \mathbb{H}$, where $k$ depends on $K$. Let

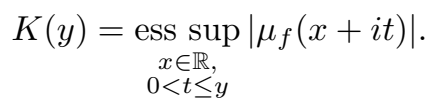

If $K(y) \searrow 0$ as $y \searrow 0$, one might expect the corresponding quasisymmetric mapping $h$ to be smoother. Carleson [5, Anderson and Hinkkanen 2, Hamilton

Received by the editors April 16, 2003 and, in revised form, September 4, 2003.

2000 Mathematics Subject Classification. Primary 30C62; Secondary 30E25.

Key words and phrases. Quasiconformal mapping, quasisymmetric mapping, Beurling-Ahlfors extension.

The author's research was supported by an FPI grant from Ministerio de Educación y Cultura (Spain) and a grant from MECD while visiting the University of Washington.

(C)2004 American Mathematical Society 
10], Semmes [14, Gardiner and Sullivan [9], Dahlberg [6], Dyn'kin [7, Belinskij and, Nikolaev and Shefel [13] among others have studied this problem.

In the remainder of the paper we will always consider $\beta$ to be a positive exponent and $n \in \mathbb{N} \cup\{0\}$ an integer such that $0<\beta-n \leq 1$.

Since we are interested in the behaviour of quasiconformal mappings near the real line, we are going to introduce the class $\mathcal{Q}_{\beta}$ that consists of those locally quasiconformal mappings for which

$$
\underset{\substack{x \in \mathbb{R}, 0<t \leq y}}{\operatorname{ess} \sup _{\substack{0 \\ 0 .}}\left|\mu_{f}(x+i t)\right|=\mathcal{O}\left(y^{\beta}\right) .}
$$

Different classes of smoothness of real functions will be used in this note.

1. Let $\Lambda^{\beta}$ stand for the class of Lipschitz functions of exponent $\beta$, i.e., those functions $h \in C^{n}(\mathbb{R})$ so that

$$
\left|h^{(n)}(x+t)-h^{(n)}(x)\right| \leq C t^{\beta-n}
$$

uniformly on compact sets.

2. We say that a function $h$ has $n$-th Peano derivative at a point $x$ if there exists a polynomial of degree at most $n, P_{n}$, such that

$$
h(x+t)-P_{n}(t)=o\left(t^{n}\right), \quad t \rightarrow 0 .
$$

Then the $n$-th Peano derivative of $h$ at $x$ is $P_{n}^{(n)}(0)$. See [15] for references. It is easy to see that if $h$ has $n$-th Peano derivative at $x(n \geq 1)$, then $h^{\prime}(x)$ exists.

We will denote by $\mathcal{P}^{\beta}$ the class of functions $h$ that can be approximated by a polynomial of degree at most $n$, in the following sense:

$$
\left|h(x+t)-P_{n}(t)\right| \leq C(x) t^{\beta} .
$$

The constant may depend on $x$, but it will be uniform in compact subsets of $\mathbb{R}$. Note that if $h \in \mathcal{P}^{\beta}$, then $h$ has $k$-th Peano derivative for any integer $k \leq \beta$. Clearly if $h \in \Lambda^{\beta}$, then $h \in \mathcal{P}^{\beta}$.

3. Finally, for $\alpha \in(0,1)$ denote by $\mathcal{M}_{\alpha}$ the collection of quasisymmetric mappings $h$ that satisfy

$$
\sup _{|x| \leq N, 0<t<N}\left|\frac{h(x+t)+h(x-t)-2 h(x)}{h(x+t)-h(x)}\right| t^{-\alpha}<\infty .
$$

This condition was introduced in more generality by Carleson in [5].

This note is a follow-up to [1] where, to answer a question raised in [2], it was shown that there are quasisymmetric Lipschitz maps of exponent $\alpha \in(0,1)$ that are not the restriction to the real line of a quasiconformal function in $\mathcal{Q}_{\alpha}$. In Theorem 2 we extend this result for any exponent $\beta>0$ and in Theorem 3 we find a sufficient condition to have locally such an extension.

\section{Dilatation DeCAY And smoothness}

In this section there will be stated several known results that relate the rate of the decay of the dilatation of a quasiconformal map in a neighbourhood of a point with the smoothness of the map at such a point.

The first of these results is the well-known Teichmüller-Wittich-Belinskij theorem that roughly asserts that when the dilatation of a quasiconformal map vanishes at 
a point, then the function is conformal at that point (see 11], pp. 224-232, for references). Namely,

Theorem A. Let $f$ be a quasiconformal map of the plane such that $f(0)=0$, $f(\infty)=\infty$ and

$$
I(r)=\frac{1}{2 \pi} \iint_{|z|<r} \frac{\left|\mu_{f}(z)\right|}{|z|^{2}} d A(z)<\infty, \quad \text { for } r<R .
$$

Then there exists a complex number $w \neq 0$ such that

$$
\left|\frac{f(z)}{z}-w\right| \leq|w| \varepsilon(|z|),
$$

where $\varepsilon(|z|) \rightarrow 0$ as $z \rightarrow 0$ and $\varepsilon$ depends only on $I, R$ and $\left\|\mu_{f}\right\|_{\infty}$.

In the same vein, the next theorem due to Nikolaev and Sheffel shows that if the dilatation decays at a certain rate in a neighbourhood of a point, then the quasiconformal map has Peano derivative at such a point (see [13]).

Theorem B. Let $f$ be quasiconformal on a disc D, centered at 0 and with radius $R$, and suppose $\left|\mu_{f}(z)\right| \leq C|z|^{\beta}$ for almost every $z$ in $D$. Then there exists a polynomial $P_{n+1}$ of degree at most $n+1,0<\beta-n \leq 1$, such that

$$
\left|f(z)-P_{n+1}(z)\right| \leq \tilde{C}|z|^{\beta+1},
$$

where $\tilde{C}$ depends on $C, \beta, R$ and the diameter of $f(D)$.

When $f$ is quasiconformal in $\mathbb{H}$ and the dilatation of $f$ decays near a boundary point, then the corresponding quasisymmetric mapping is smooth at that point. This was Carleson's point of view in [5. Concretely,

Theorem C. Let $\alpha \in(0,1)$. Then $f \in \mathcal{Q}_{\alpha}$ if and only if $h=\left.f\right|_{\mathbb{R}} \in \mathcal{M}_{\alpha}$.

The theorem appears in this form in [1].

For general exponents $\beta>0$, something else can be said about the smoothness of the quasisymmetric restriction, as the following theorem due to Anderson and Hinkkanen and Dyn'kin shows.

Theorem D. If $f \in \mathcal{Q}_{\beta}$, then $h=\left.f\right|_{\mathbb{R}} \in \Lambda^{\beta+1}$.

Anderson and Hinkkanen have obtained that $h \in \Lambda^{\gamma}$ for all $\gamma<\beta+1$ (see [2]). Dyn'kin showed the result in the form presented here (see [7, Theorem 5).

\section{Quasiconformal extensions}

Here and hereafter by a quasiconformal extension we mean a quasiconformal extension to the upper half-plane of a quasisymmetric mapping of the real line that fixes $\infty$.

In the middle 1950s Beurling and Ahlfors showed that

$$
f(x+i y)=\frac{1}{2}\left(\int_{0}^{1} h(x+t y)+h(x-t y) d t\right)+\frac{i}{2}\left(\int_{0}^{1} h(x+t y)-h(x-t y) d t\right)
$$

is a quasiconformal extension of $h$ whenever $h$ is a quasisymmetric mapping.

Many variants of the Beurling-Ahlfors construction have been studied where the real part of the extension consists of a convolution of $h$ with a positive even kernel 
$K_{e}$ that integrates to 1 and whose imaginary part is a convolution with an odd kernel, $K_{o}$ such that $K_{o}(x) \geq 0$ for $x \geq 0$. In order to obtain a quasiconformal extension, decay conditions on the kernels as $|x| \rightarrow \infty$ are needed (see, for example, [8], p. 115).

In the same line we can consider extensions defined as follows. Let $h: \mathbb{R} \rightarrow \mathbb{R}$ be an increasing homeomorphism, $h \in C^{1}(\mathbb{R})$. We say that $h \in \mathcal{A} B_{k}$ if there exist $k+1$ distinct real numbers $0<a_{0}<\ldots<a_{k}<1$ and $M \geq 1$ so that

$$
\frac{1}{M} \leq \frac{\sum_{j=0}^{k} h^{\prime}\left(x+a_{j} y\right)}{\sum_{j=0}^{k} h^{\prime}\left(x-a_{j} y\right)} \leq M
$$

for every $x \in \mathbb{R}$ and $y \geq 0$.

Theorem 1. $h \in \mathcal{A} B_{k}$ if and only if

$$
\begin{aligned}
f(x+i y) & =\frac{1}{2}\left(\sum_{j=0}^{k} p_{j}\left(h\left(x+a_{j} y\right)+h\left(x-a_{j} y\right)\right)\right) \\
& +\frac{i}{2}\left(\sum_{j=0}^{k} p_{j}\left(h\left(x+a_{j} y\right)-h\left(x-a_{j} y\right)\right)\right)
\end{aligned}
$$

is a quasiconformal extension of $h$, where $a_{j} \in(0,1)$ as given above and the $p_{j}$ are positive numbers such that $\sum_{j=0}^{k} p_{j}=1$.

Here we are considering $\frac{1}{2} \sum_{j=0}^{k} p_{j}\left(\delta_{a_{j}}+\delta_{-a_{j}}\right)$ as the even kernel of the extension and $\frac{1}{2} \sum_{j=0}^{k} p_{j}\left(\delta_{a_{j}}-\delta_{-a_{j}}\right)$ as the odd kernel.

Remarks.

1. If the condition $\mathcal{A} B_{k}$ is defined locally (i.e., the quotient is locally bounded), then the extension is locally quasiconformal.

2. $h \in \mathcal{A} B_{0}$ if and only if $h$ is a $C^{1}(\mathbb{R})$ homeomorphism such that $\log h^{\prime}$ is bounded. As a consequence, $h$ is a $C^{1}(\mathbb{R})$ increasing bi-Lipschitz homeomorphism.

3. If $h \in \mathcal{A} B_{k}$, then $h$ is quasisymmetric.

4. Choosing $a_{j}$ so that $\frac{1}{2 k}<\left|a_{j}-a_{j-1}\right| \leq \frac{2}{k}$ and taking limits in the extension one gets the Beurling-Ahlfors extension.

Proof. Clearly $f$ is an extension of $h$, i.e., $\left.f\right|_{\mathbb{R}}=h$. Indeed, $f(x)=\frac{1}{2} \sum_{j=0}^{k} p_{j} 2 h(x)$ $=h(x) \sum_{j=0}^{k} p_{j}=h(x)$.

Notice also that $f$ is a proper function, that is, $\lim _{|z| \rightarrow \infty}|f(z)|=\infty$.

The modulus of $f$ is given by

$$
2|f|^{2}=\left(\sum_{j=0}^{k} p_{j} h\left(x+a_{j} y\right)\right)^{2}+\left(\sum_{j=0}^{k} p_{j} h\left(x-a_{j} y\right)\right)^{2} .
$$

Let $z=x+i y$. Then, if $|z| \rightarrow \infty$, either $x+a_{j} y \rightarrow \infty$ for all $j$, or $x-a_{j} y \rightarrow-\infty$ for all $j$. Since every $p_{j}$ is positive, $|f| \rightarrow \infty$; i.e., $f$ is proper. 
Next, it will be shown that $f: \mathbb{H} \rightarrow \mathbb{H}$. Since $h$ is an increasing homeomorphism and the $a_{j}$ 's and the $p_{j}$ 's are all positive, we get

$$
\sum_{j} p_{j} h\left(x+a_{j} y\right)>\sum_{j} p_{j} h\left(x-a_{j} y\right)
$$

and therefore $\operatorname{Im}(f)>0$. This shows $f: \mathbb{H} \rightarrow \mathbb{H}$.

Since $f$ is proper, to show that $f$ is a homeomorphism it suffices to show that $f$ is injective. For $k=0$, this follows easily from the injectivity of $h$. Suppose $k \geq 1$.

Observe that $f$ is injective in the upper half-plane if and only if

$$
\sum_{j=0}^{k} p_{j} h\left(x+a_{j} y\right)=\sum_{j=0}^{k} p_{j} h\left(x^{\prime}+a_{j} y^{\prime}\right) \Rightarrow \sum_{j=0}^{k} p_{j} h\left(x-a_{j} y\right) \neq \sum_{j=0}^{k} p_{j} h\left(x^{\prime}-a_{j} y^{\prime}\right),
$$

for $x+i y \neq x^{\prime}+i y^{\prime}$ and $y, y^{\prime}>0$.

Suppose

$$
\sum_{j=0}^{k} p_{j} h\left(x+a_{j} y\right)=\sum_{j=0}^{k} p_{j} h\left(x^{\prime}+a_{j} y^{\prime}\right) .
$$

If $h\left(x+a_{j} y\right)=h\left(x^{\prime}+a_{j} y^{\prime}\right)$ for every $j$ we would have $x=x^{\prime}$ and $y=y^{\prime}$, since $h$ is a homeomorphism, and $k \geq 1$. Thus, there exist $l$ and $m$ such that

$$
h\left(x+a_{l} y\right)<h\left(x^{\prime}+a_{l} y^{\prime}\right) \quad \text { and } \quad h\left(x+a_{m} y\right)>h\left(x^{\prime}+a_{m} y^{\prime}\right) .
$$

Assume $a_{l}<a_{m}$. Since $h$ is increasing, the points are ordered as

$$
x+a_{l} y<x^{\prime}+a_{l} y^{\prime}<x^{\prime}+a_{m} y^{\prime}<x+a_{m} y,
$$

which implies that $x<x^{\prime}$ and $y>y^{\prime}>0$ and so, $x-a_{j} y<x^{\prime}-a_{j} y^{\prime}$ for all $j$. Using again that $h$ is monotone and that each $p_{j}$ is positive,

$$
\sum_{j=0}^{k} p_{j} h\left(x-a_{j} y\right)<\sum_{j=0}^{k} p_{j} h\left(x^{\prime}-a_{j} y^{\prime}\right) .
$$

So $f$ is injective.

Finally, we shall see that the dilatation of $f$ is bounded away from 1 , that is, $\left|\mu_{f}(z)\right| \leq \kappa<1$. Doing some computations we get

$$
\begin{aligned}
4 f_{z}(x, y) & =\sum_{j=0}^{k} p_{j}\left(\left(1+a_{j}\right)+i\left(1-a_{j}\right)\right) h^{\prime}\left(x+a_{j} y\right) \\
& +\sum_{j=0}^{k} p_{j}\left(\left(1+a_{j}\right)-i\left(1-a_{j}\right)\right) h^{\prime}\left(x-a_{j} y\right), \\
4 f_{\bar{z}}(x, y) & =\sum_{j=0}^{k} p_{j}\left(\left(1-a_{j}\right)+i\left(1+a_{j}\right)\right) h^{\prime}\left(x+a_{j} y\right) \\
& +\sum_{j=0}^{k} p_{j}\left(\left(1-a_{j}\right)-i\left(1+a_{j}\right)\right) h^{\prime}\left(x-a_{j} y\right) .
\end{aligned}
$$


Now setting

$$
\begin{aligned}
& A=\sum_{j=0}^{k} p_{j}\left(1+a_{j}\right) h^{\prime}\left(x+a_{j} y\right), \quad B=\sum_{j=0}^{k} p_{j}\left(1+a_{j}\right) h^{\prime}\left(x-a_{j} y\right), \\
& C=\sum_{j=0}^{k} p_{j}\left(1-a_{j}\right) h^{\prime}\left(x+a_{j} y\right), \quad D=\sum_{j=0}^{k} p_{j}\left(1-a_{j}\right) h^{\prime}\left(x-a_{j} y\right),
\end{aligned}
$$

we have that $4 f_{z}=(A+B)+i(C-D)$ and $4 f_{\bar{z}}=(C+D)+i(A-B)$ and then the expression for the dilatation of $f$ becomes

$$
\mu_{f}=\frac{(C+D)+i(A-B)}{(A+B)+i(C-D)} .
$$

Now if $h \in \mathcal{A} B_{k}$, then

$$
\frac{1}{\tilde{M}} \leq \frac{A}{B} \leq \tilde{M} .
$$

Observe that $\tilde{M}$ could depend on $p_{j}$ and $a_{j}$.

Clearly $A>C$ and $B>D$. So there exists a $\lambda<1$ (depending only on $a_{j}$, $j=0, \ldots, n)$ such that $\lambda A>C$ and $\lambda B>D$. Therefore,

$$
A B-C D>\delta\left(A^{2}+B^{2}+C^{2}+D^{2}\right)
$$

where $\delta$ depends on $M$ and $\lambda$. We conclude that

$$
\left|\mu_{f}(z)\right|^{2}=\frac{A^{2}+B^{2}+C^{2}+D^{2}-2(A B-C D)}{A^{2}+B^{2}+C^{2}+D^{2}+2(A B-C D)} \leq \kappa^{2}<1,
$$

for some $\kappa$ that depends on $M$ and $\lambda$.

Conversely, if

$$
\left|\mu_{f}(z)\right| \leq \kappa<1
$$

then there exists $\delta>0$ so that

$$
A B-C D>\delta\left(A^{2}+B^{2}+C^{2}+D^{2}\right) .
$$

Since $\lambda A>C$ and $\lambda B>D$ for some $\lambda<1$, we get

$$
\frac{1}{M} \leq \frac{A}{B} \leq M,
$$

where $M$ depends on $\delta$ and $\lambda$. Therefore $h \in \mathcal{A} B_{k}$.

Observe that if we write $f(z)=u(z)+i v(z)$, then $A B-C D>\delta\left(A^{2}+B^{2}+C^{2}+\right.$ $\left.D^{2}\right)$ is equivalent to $u_{x}(z) v_{y}(z)-v_{x}(z) u_{y}(z)>\delta\left(u_{x}(z)^{2}+v_{x}(z)^{2}+u_{y}(z)^{2}+v_{y}(z)^{2}\right)$.

\section{Converse Results}

In [1, it was shown by a complicated argument that Theorem $\mathrm{D}$ does not have a converse for $\beta<1$. Next we will show that the result is true for any positive $\beta$.

Theorem 2. For each $\beta>0$ there exists a quasisymmetric homeomorphism, $h$ : $\mathbb{R} \rightarrow \mathbb{R}$ in $\Lambda^{\beta}$ that cannot be extended to a quasiconformal map in $\mathcal{Q}_{\gamma}$, for any $\gamma>0$.

To prove Theorem 2 it suffices to find a quasisymmetric map, $h$, that is in $\Lambda^{\beta}$ for $\beta>0$ and is not in $\mathcal{M}_{\alpha}$ for any $\alpha \in(0,1)$. Theorem $[$ implies that there does not exist an extension of $h$ in $\mathcal{Q}_{\gamma}$ for any $\gamma>0$, which would conclude the proof of Theorem 2 So it is enough to show the following lemma.

Lemma 1. For $n>1, h(x)=x|x|^{n-1}$ is a quasisymmetric homeomorphism that belongs to $\Lambda^{n}$, but it is not in $\mathcal{M}_{\alpha}$ for any $\alpha \in(0,1)$. 
Proof. First, $f(z)=z|z|^{n-1}$ is a quasiconformal extension of $h$ to $\mathbb{H}$; therefore, $h$ is quasisymmetric.

Now choose $x=t$. Then

$$
\left|\frac{h(x+t)+h(x-t)-2 h(x)}{h(x+t)-h(x)}\right|=\left|\frac{2 t|2 t|^{n-1}-2 t|t|^{n-1}}{2 t|2 t|^{n-1}-t|t|^{n-1}}\right|=\frac{2^{n}-2}{2^{n}-1},
$$

and therefore, $h \notin \mathcal{M}_{\alpha}$ for any $\alpha \in(0,1)$.

Finally, $h \in \Lambda^{n}$, since $h^{(n-1)}(x)=n ! x$ if $n$ is odd and $h^{(n-1)}(x)=n !|x|$ if $n$ is even.

Although Theorem $\mathrm{D}$ does not admit a converse, with some different conditions a certain converse can be proved.

Theorem 3. Let $f \in \mathcal{Q}_{\beta}$ and $h=\left.f\right|_{\mathbb{R}}$. Then $\log h^{\prime}$ is continuous on $\mathbb{R}$ and $h \in \mathcal{P}^{\beta+1}$.

Conversely, let $h$ be an increasing homeomorphism such that $h^{\prime} \in \mathcal{P}^{\beta}$ and $\log h^{\prime}$ is locally bounded. Then for every $x \in \mathbb{R}$ there exists a neighbourhood of $x, U_{x} \subset \mathbb{R} \cup \mathbb{H}$ and an extension $f$ of $h$ in $\mathbb{H}$ that is quasiconformal in $U_{x}$ and such that

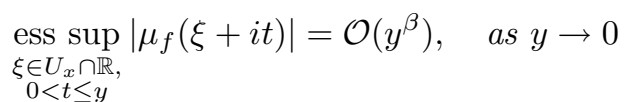

for $y$ so that $x+i y \in U_{x}$.

Notice that the direct part of this theorem is a consequence of Theorem D Nevertheless, the proof presented here uses different techniques.

Proof. The direct part of Theorem 3 is a corollary of Theorems $\mathrm{A}$ and B of $\S 1$.

First we will see that $\log h^{\prime}$ is continuous as a consequence of Theorem Indeed, by Carleson's result (see [5], p. 1), $h^{\prime}$ is a continuous function on $\mathbb{R}$. So there remains only to show that $h^{\prime}(x) \neq 0$ for all $x \in \mathbb{R}$.

Fix $x \in \mathbb{R}$ and define

$$
F_{x}(z)= \begin{cases}f(z+x)-h(x), & z \in \mathbb{H} \cup \mathbb{R}, \\ \bar{f}(\bar{z}+x)-h(x), & z \in \mathbb{H}^{-},\end{cases}
$$

where $\mathbb{H}^{-}=\{z: \operatorname{Im}(z)<0\}$. Then the mapping $F_{x}(z)$ is locally quasiconformal, and it fixes 0 and $\infty$. It has complex dilatation

$$
\mu_{F_{x}}(z)= \begin{cases}\mu_{f}(x+z), & z \in \mathbb{H} \cup \mathbb{R}, \\ \mu_{\bar{f}}(x+\bar{z}), & z \in \mathbb{H}^{-} .\end{cases}
$$

So $\left|\mu_{F_{x}}(z)\right| \leq C|z|^{\beta}$ in a neighbourhood of zero (since $f \in \mathcal{Q}_{\beta}$ ).

Therefore $F_{x}(z)$ satisfies the hypothesis of Theorem $\mathrm{A}$. So there exists a complex number, $w_{x} \neq 0$, such that

$$
\left|\frac{F_{x}(z)}{z}-w_{x}\right| \leq\left|w_{x}\right| \varepsilon(|z|) .
$$

Now, for $t \in \mathbb{R}$

$$
\lim _{t \rightarrow 0} \frac{F_{x}(t)}{t}=\lim _{t \rightarrow 0} \frac{h(x+t)-h(x)}{t}=h^{\prime}(x),
$$

and so $h^{\prime}(x)=w_{x} \neq 0$.

Using Theorem $\mathrm{B}$ we will find a polynomial that approximates $h$. Again, fix $x \in$ $\mathbb{R}$, and define $G_{x}(z)=F_{x}(z) / h^{\prime}(x)$. Since $h^{\prime}(x) \neq 0, G_{x}(z)$ is a local quasiconformal 
mapping in $\mathbb{C}$ fixing 0 and $\infty$ and $\frac{\partial G_{x}}{\partial z}(0)=1$. Moreover, $G_{x}(z)$ has complex dilatation, $\left|\mu_{G_{x}}(z)\right| \leq C|z|^{\beta}$ in some disc $D(0, R)$ where $R$ is uniform on compact sets since $f \in \mathcal{Q}_{\beta}$.

By Theorem $\mathrm{A}$ we get

$$
\left|G_{x}(z)\right| \leq|z|(1+\varepsilon(|z|))
$$

where $\varepsilon$ depends on $I, R$ and $\left\|\mu_{G_{x}}\right\|_{\infty}$. Since $\left\|\mu_{G_{x}}\right\|_{\infty}=\left\|\mu_{f}\right\|_{\infty}$ and $R$ is uniform on compact sets, there exists a uniform $\rho>0$, small enough so that

$$
\left|G_{x}(z)\right| \leq C|z|, \quad \text { for }|z|<\rho .
$$

Then,

$$
\operatorname{diam}\left(G_{x}(D(0, \rho / 2)) \leq C,\right.
$$

$C$ uniform on compact sets. Note that neither $\rho$ nor $C$ depend on $x$ since the decay condition on $\mu_{f}$ is uniform on compact subsets of the real line.

By Theorem B, there exists a polynomial $Q_{x}^{n+1}(z)$ such that

$$
\left|G_{x}(z)-Q_{x}^{n+1}(z)\right| \leq \tilde{C}|z|^{\beta+1}
$$

where $\tilde{C}=\tilde{C}(C, \beta)$ does not depend on $x$, and therefore it is uniform on compact sets. In particular, for $z=t \in \mathbb{R}^{+}, t$ small enough,

$$
\left|h(x+t)-P_{x}^{n+1}(t)\right| \leq \tilde{C}\left|h^{\prime}(x)\right| t^{\beta+1},
$$

uniformly on compact sets, where $P_{x}^{n+1}(t)=h(x)+h^{\prime}(x) Q_{x}^{n+1}(t)$.

To prove the converse direction, as usual, let $n$ be chosen so that $0<\beta-n \leq 1$ and consider the function

$$
A(x+i y)=\sum_{j=0}^{n+1} p_{j} h\left(x+a_{j} y\right),
$$

where $a_{j} \in(0,1)$ for $j \in\{0, \ldots, n+1\}$. Choose $p_{j}, j \in\{0, \ldots, n+1\}$, so that

$$
\sum_{j=0}^{n+1} p_{j} a_{j}^{k}=(-1)^{[k / 2]}, \quad \text { for } k=0, \ldots, n+1,
$$

where $[x]$ denotes the integer part of $x$. Notice that (2) defines a linear system of $n+2$ equations and $n+2$ unknowns, with the Vandermonde matrix as the coefficient matrix of the system.

As in Theorem 1, define the extension of $h$ given by

$$
f(x+i y)=\frac{1}{2}(A(x+i y)+A(x-i y))+\frac{i}{2}(A(x+i y)-A(x-i y)) .
$$

In this case, condition (2) forces some of the $p_{j}$ 's to be negative, and therefore we cannot conclude that $f$ is a homeomorphism.

First, we shall show that for each $x \in \mathbb{R}$, there exists a neighbourhood of $x$, $V_{x} \subset \mathbb{R} \cup \mathbb{H}$ such that $\left|f_{z}(z)\right|>0$ for $z \in V_{x}$. 
Since $h \in C^{1}(\mathbb{R})$, the expression for $f$ can be differentiated, and evaluating at $z=x+i y$,

$$
\begin{aligned}
& 4 f_{\bar{z}}(x+i y)=\sum_{j=0}^{n+1} w_{j} h^{\prime}\left(x+a_{j} y\right)+\bar{w}_{j} h^{\prime}\left(x-a_{j} y\right), \\
& 4 f_{z}(x+i y)=i \sum_{j=0}^{n+1} \bar{w}_{j} h^{\prime}\left(x+a_{j} y\right)-w_{j} h^{\prime}\left(x-a_{j} y\right)
\end{aligned}
$$

where $w_{j}=p_{j}\left(\left(1-a_{j}\right)+i\left(\left(1+a_{j}\right)\right)\right.$.

For $y=0$, condition (2) on the $a_{j}$ 's and $p_{j}$ 's implies

$$
4 f_{z}(x)=-i \sum_{j}\left(w_{j}-\bar{w}_{j}\right) h^{\prime}(x)=2 \sum_{j} p_{j}\left(1+a_{j}\right) h^{\prime}(x)=4 h^{\prime}(x) \neq 0,
$$

since $\log h^{\prime}$ is locally bounded in $\mathbb{R}$.

On the other hand, condition (21) yields

$$
4 f_{\bar{z}}(x)=\sum_{j}\left(w_{j}+\bar{w}_{j}\right) h^{\prime}(x)=2 \sum_{j} p_{j}\left(1-a_{j}\right) h^{\prime}(x)=2(1-1) h^{\prime}(x)=0 .
$$

Therefore,

$$
J_{f}(x)=\left|f_{z}(x)\right|^{2}-\left|f_{\bar{z}}(x)\right|^{2}=\left(h^{\prime}(x)\right)^{2}>0
$$

where $J_{f}$ denotes the Jacobian of $f$. Since $J_{f}$ is continuous $\left(h\right.$ is a $C^{1}(\mathbb{R})$ homeomorphism) there exists a neighbourhood of $x, V_{x}$, so that $J_{f}(z)>0$ in $V_{x}$. In particular, $\left|f_{z}(z)\right|>0$ for every $z \in V_{x}$.

Next we show that there exists a neighbourhood of $x, U_{x}$, such that

$$
\underset{\substack{\text { ess } \\ \xi \in U_{x} \cap \mathbb{R}, t \leq y}}{\sup }\left|\mu_{f}(\xi+i t)\right|=\mathcal{O}\left(y^{\beta}\right)
$$

for $y$ such that $\xi+i y \in U_{x}$.

Since $h^{\prime} \in \mathcal{P}^{\beta}$ there exists a polynomial of degree at most $n, P_{n}^{x}(t)$, that approximates $h^{\prime}(x+t)$ for small $t$. Write $P_{n}^{x}(t)=\sum_{m=0}^{n} c_{m}(x) t^{m}$ where $c_{0}(x)=h^{\prime}(x)$. Take $U_{x} \subset V_{x}, x \in U_{x}$, such that for any $\xi+i t \in U_{x}$ the polynomial approximation of $h^{\prime}$ exists at $\xi+a_{j} t, j=0, \ldots, n+1$.

Let $\xi+i t=x+s+i t \in U_{x}$. So if we replace $h^{\prime}$ by its approximation, then

$$
\begin{aligned}
4 f_{\bar{z}}(\xi+i t) & =\sum_{j=0}^{n+1} w_{j} h^{\prime}\left(\xi+a_{j} t\right)+\bar{w}_{j} h^{\prime}\left(\xi-a_{j} t\right) \\
& =\sum_{j=0}^{n+1} w_{j}\left(\sum_{m=0}^{n} c_{m}(x)\left(s+a_{j} t\right)^{m}\right) \\
& +\sum_{j=0}^{n+1} \bar{w}_{j}\left(\sum_{m=0}^{n} c_{m}(x)\left(s-a_{j} t\right)^{m}\right)+C(x) \mathcal{O}\left(t^{\beta}\right) \\
& =\sum_{m=0}^{n} \sum_{k=0}^{m}\left(\sum_{j=0}^{n+1}\left(w_{j}+(-1)^{k} \bar{w}_{j}\right) a_{j}^{k}\right)\left(\begin{array}{c}
m \\
k
\end{array}\right) c_{m}(x) t^{k} s^{m-k} \\
& +C(x) \mathcal{O}\left(t^{\beta}\right) .
\end{aligned}
$$

To calculate $\sum_{j=0}^{n+1}\left(w_{j}+(-1)^{k} \bar{w}_{j}\right) a_{j}^{k}, k=0, \ldots, n$, we consider separately the cases of even and odd $k$. 
- For even $k$, observe that $[k / 2]=[(k+1) / 2]$. By condition (2),

$$
\sum_{j=0}^{n+1}\left(w_{j}+\bar{w}_{j}\right) a_{j}^{k}=2 \sum_{j} p_{j}\left(1-a_{j}\right) a_{j}^{k}=2\left((-1)^{[k / 2]}-(-1)^{[(k+1) / 2]}\right)=0 .
$$

- For odd $k$, observe that $[k / 2]+1=[(k+1) / 2]$. By condition (2),

$$
\sum_{j=0}^{n+1}\left(w_{j}-\bar{w}_{j}\right) a_{j}^{k}=2 i \sum_{j} p_{j}\left(1+a_{j}\right) a_{j}^{k}=2 i\left((-1)^{[k / 2]}+(-1)^{[(k+1) / 2]}\right)=0 .
$$

Therefore, for every $\xi+i t \in U_{x}$,

$$
4 f_{\bar{z}}(\xi+i t)=C(x) \mathcal{O}\left(t^{\beta}\right) .
$$

Analogously, for $f_{z}$,

$$
\begin{aligned}
4 f_{z}(\xi+i t) & =i \sum_{j=0}^{n+1} \bar{w}_{j} h^{\prime}\left(\xi+a_{j} t\right)-w_{j} h^{\prime}\left(\xi-a_{j} t\right) \\
& =i \sum_{m=0}^{n} \sum_{k=0}^{m}\left(\sum_{j=0}^{n+1}\left(\bar{w}_{j}+(-1)^{k+1} w_{j}\right) a_{j}^{k}\right)\left(\begin{array}{c}
m \\
k
\end{array}\right) t^{k} s^{m-k} c_{m}(x) \\
& +C(x) \mathcal{O}\left(t^{\beta}\right) \\
& =\sum_{k=0}^{n} B_{k}(x, s) t^{k}+C(x) \mathcal{O}\left(t^{\beta}\right),
\end{aligned}
$$

where $B_{k}(x, s)=4 b_{k} \sum_{m=k}^{n}\left(\begin{array}{c}m \\ k\end{array}\right) c_{m}(x) s^{m-k}$ and $b_{k}= \begin{cases}(-1)^{[k / 2]}, & \text { if } k \text { is even, } \\ i(-1)^{[k / 2]}, & \text { if } k \text { is odd. }\end{cases}$

Observe that, for every $k, B_{k}(x, s)$ is continuous in $s$ and $B_{0}(x, 0)=c_{0}(x)=$ $h^{\prime}(x) \neq 0$.

So the dilatation of $f$ at $\xi+i t \in U_{x}$ is

$$
\mu_{f}(\xi+i t)=\frac{C(x) \mathcal{O}\left(t^{\beta}\right)}{\sum_{k=0}^{n} B_{k}(x, s) t^{k}+C(x) \mathcal{O}\left(t^{\beta}\right)},
$$

and thus,

$$
\underset{\substack{\text { ess } \\ \xi \in U_{x} \cap \mathbb{R} \\ t \leq y}}{\operatorname{enp}}\left|\mu_{f}(\xi+i t)\right|=\mathcal{O}\left(y^{\beta}\right) \text {. }
$$

Remarks.

1. In this case, in order to obtain (2), we need some $p_{j}$ to be negative. In Theorem 1 it was necessary to require each $p_{j}$ to be positive in order to obtain a homeomorphic extension.

2. This technique of cancelling the intermediate derivatives of a function by adding up the values of the function evaluated at different points is known as Richardson's extrapolation method. See [3], p. 372 and [12], p. 194.

3. Notice that if $h \in \Lambda^{\beta}$ and $\log h^{\prime}$ is continuous, then $h^{\prime} \in \mathcal{P}^{\beta}$, and therefore there exists a local extension of $h$ in $\mathcal{Q}_{\beta}$. 


\section{ACKNOWLEDGMENT}

The results of this note were part of my doctoral thesis at the Universidad Autónoma de Madrid. I would like to express my gratitude to my advisor José Luis Fernández for many enlightening discussions and to Milne Anderson for posing the question that stimulated this investigation.

\section{REFERENCES}

[1] J.M. Anderson, A. Cantón and J.L. Fernández, On smoothness of symmetric mappings, Complex Var. Theory Appl., 37 (1998), 161-169.

[2] J.M. Anderson and A. Hinkkanen, Quasiconformal selfmappings with smooth boundary values, Bull. London Math. Soc., 26 (1994), 549-556. MR96a:30018

[3] K.E. Atkinson, An Introduction to Numerical Analysis, Second edition, John Wiley \& Sons, Inc., New York (1989). MR90m:65001

[4] A. Beurling and L.V. Ahlfors, The boundary correspondence under quasiconformal mappings, Acta Math., 96 (1956), 125-142. MR 19:258c

[5] L. Carleson, On mappings, conformal at the boundary, J. Analyse Math., 19 (1967), 1-13. MR.35:6821

[6] B.E.J. Dahlberg, On the absolute continuity of elliptic measures, Amer. J. Math., 108 (1986), 1119-1138. MR88i:35061

[7] E. Dyn'kin, Estimates for asymptotically conformal mappings, Ann. Acad. Sci. Fenn. Math., 22 (1997), no. 2, 275-304. MR.98h:30030

[8] R.A. Fefferman, C.E. Kenig and J. Pipher, The theory of weights and the Dirichlet problem for elliptic equations, Ann. of Math. (2), 134 (1991), 65-124. MR93h:31010

[9] F.P. Gardiner and D.P. Sullivan, Symmetric structures on a closed curve, Amer. J. Math., 114 (1992), 683-736. MR95h:30020

[10] D.H. Hamilton, Rectifiable Julia curves, J. London Math. Soc. (2), 54 (1996), no. 3, 530-540. MR 98j:30022

[11] O. Lehto and K.I. Virtanen, Quasiconformal Mappings in the Plane. Second edition. SpringerVerlag, New York-Heidelberg (1973). MR 49:9202

[12] D.G. Moursund and C.S. Duris, Elementary Theory and Applications of Numerical Analysis, Dover Publications, Inc., New York (1988). MR92a:65006

[13] I.G. Nikolaev and S.Z. Shefel, Differential properties of mappings that are conformal at a point, Siberian Math. J., 27, 1, (1986), 106-114. MF87i:30037

[14] S. Semmes, Quasiconformal mappings and chord-arc curves, Trans. Amer. Math. Soc., 306, (1988), 233-263. MR 89j:30029

[15] C.E. Weil, The Peano derivative: what's known and what isn't, Real Anal. Exchange, 9, (1983/84), no. 2, 354-365. MF 86c:26007

Department of Mathematics, University of Washington, Box 354350, Seattle, WashINGTON 98195-4350

Current address: Departament de Matemàtiques, Universitat Autònoma de Barcelona, 08193 Bellaterra (Barcelona), Spain

E-mail address: acanton@mat.uab.es 